\title{
Énfasis y orden de palabras en algunas construcciones con vaya ${ }^{1}$
}

Palabras clave: interjección, término terciario, foco, énfasis, intensificación, gramática

La forma del subjuntivo vaya se ha ido diversificando en español y ha dado lugar a unidades de distinta categoría y función, tal como muestran los ejemplos siguientes:

(1) Mi deseo es que se vaya pronto.

(2) No sé cuándo volverá, vaya usted a saber.

(3) ¿Ya estás aquí? ¡Vaya, qué sorpresa!

(4) Volvieron a ganar. Lo de siempre, vaya.

(5) Vaya pinta tiene ese...; Vaya susto que me has dado...

(6) Me ubiqué en la vida diaria, jvaya si me ubiqué!; Y te dejas, vaya que si te dejas.

Nos encontramos, pues, con casos en que vaya se realiza como verbo pleno (1), con expresiones formularias con un alto grado de fijación en las que la unidad ha ido abandonando ya cualquier rasgo de movimiento a favor de contenidos de duda o incertidumbre (2), con usos que convertirían esta unidad en una interjección (3), con otros que parecen oscilar entre la interjección y los denominados «marcadores discursivos» (4) y, finalmente, con las secuencias de

1 Este trabajo se incluye en la investigación desarrollada en el proyecto «Gramática y discurso: procedimientos lingüísticos de la interacción comunicativa», financiado por el Ministerio de Economía y Competitividad del Gobierno de España (FFI2013-41323-P). 
(5) y (6), cuya frontera con los sintagmas interjectivos no parece delimitada con nitidez ${ }^{2}$.

Nos centraremos aquí en el análisis de los ejemplos de (6), aunque se aludirá también a los anteriores para establecer aquellos rasgos que tienen en común o con los que contrastan. Trataremos de probar: $a$ ) que en estos casos vaya no tiene categoría de interjección, y por lo tanto no funciona como núcleo de la construcción ni el segmento que le sigue constituye su adyacente; $b$ ) que se trata de una unidad cuya función semántica no es otra que la de aportar una intensificación o gradación máxima al grupo de categoría verbal (en otros casos, nominal) al que se subordina desde una posición sintagmática prenuclear; c) que las conjunciones si y que no actúan como transpositores de la oración a categoría nominal, sino que forman parte de la expresión cuantificadora por resultar requeridas cuando lo que se intensifica es un predicado.

En primer lugar $(\$ 1)$, se expondrán los requisitos formales que permiten caracterizar a los sintagmas interjectivos y las similitudes y diferencias que estos mantienen con los enunciados exclamativos. A continuación $(\$ 2)$, se señalarán los rasgos de la construcción de término terciario en español con el fin de justificar que las secuencias de vaya si \{vaya que, vaya que si $\}$ constituyen adyacentes oracionales que reúnen estas propiedades. Seguidamente $(\$ 3)$, se analizarán las variantes que presenta esta construcción, contrastándola con otras con las que comparte el rasgo de 'intensificación'. Por último (\$4), se explora la posibilidad de que el español albergue en su estructura gramatical un morfema de 'énfasis'.

2 La última edición del diccionario de la Academia (RAE 2014) anota dos categorías gramaticales bajo el lema vaya: de un lado, como interjección en usos como Pablo ba aprobado todas las asignaturas, ;vaya!; No podemos ir al teatro: se ba suspendido la sesión, jvaya!; ;Vaya con el niño!; de otro, como adjetivo exclamativo: ;Vaya películas veis!; ¡Vaya reloj tienes!; ¡Vaya fuerza tuvieron que hacer! (DRAE, s.v. vaya). Por su parte, la NGLE (RAE y ASALE 2009, \$32.2.c) considera además grupos sintácticos interjectivos aquellos «constituidos por las interjecciones y diversos complementos que las acompañan, como en caramba con la niña, cuidado con el perro o vaya con el viejito». Incluye asimismo dentro de la interjección ( $\$ 32.2 . \mathrm{i})$ unidades como vaya, bueno, vale cuando se presentan duplicadas (vaya, vaya; bueno, bueno...), lo que difumina las fronteras entre los sintagmas interjectivos y las unidades que se utilizan como marcadores discursivos. 


\section{La frontera entre las interjecciones y los enunciados exclamativos}

\subsection{La categoría interjectiva}

Para la corriente del funcionalismo español en la que nos inscribimos ${ }^{3}$, las categorías se definen teniendo en cuenta su capacidad de combinatoria sintagmática, sobre la base de las relaciones hjelmslevianas entre las unidades (Hjelmslev 1943: 64; Martínez 1994a: \$ 1.9, 1.17, 2.3.6, 4.3; 1994b: \$ 1.3.1). Así, a partir de la relación de dependencia pueden establecerse dos grandes categorías de sintagmas: verbales y nominales; y dentro de estos, sintagmas sustantivos, adjetivos y adverbiales ${ }^{4}$. Se considera, además, una quinta categoría, la de los sintagmas interjectivos, establecida a partir de la relación de combinación: estos quedan definidos por la simple compatibilidad, en forma de inciso, con el resto de categorías (Martínez 1994a: 99).

La interjección presenta, pues, un estatus peculiar, que se refleja en algunos aspectos. En primer lugar $-\mathrm{y}$ a diferencia del resto de los sintagmas-, no puede entrar en relaciones de combinación o subordinación con otras categorías para formar grupos sintagmáticos (López Bobo 2002: 16-17), pues su capacidad de relación queda limitada a la simple yuxtaposición. Más aun, esta se presenta de forma restrictiva si partimos del hecho de que la relación de yuxtaposición admite, generalmente, ser semantizada por la presencia de un coordinador, algo vedado a los sintagmas interjectivos: ${ }_{i}^{*}$ Uy y vaya!; En segundo lugar, mientras que los verbos, sustantivos, adjetivos o adverbios admiten ser transpuestos a otras categorías, «ningún sintagma puede transponerse a categoría interjectiva» (Martínez 1994a: 104). En tercer lugar, constituyen signos autónomos mínimos en cuyo interior no es posible detectar la presencia de signo léxico y morfológico. En cuarto lugar, carecen de función referencial específica, esto es, se trata de signos privados de la capacidad de remitir a

3 Una exposición sintética de los principios del funcionalismo de corte alarquiano y, en particular, de las aportaciones desarrolladas en la Universidad de Oviedo, puede hallarse en San Julián Solana (2014).

4 A partir de la relación glosemática de selección (equiparable a la tradicional subordinación), se definen los dos grandes grupos de categorías: los sintagmas verbales, que salvo existencia de transposición siempre funcionan como núcleo de los sintagmas nominales, y estos como adyacentes suyos. La misma relación permite establecer subcategorías entre los sintagmas nominales: el sustantivo siempre funciona como núcleo del adjetivo, y este como adyacente suyo; el adjetivo siempre funciona como núcleo del adverbio, y este como su adyacente. Los adverbios pueden subordinarse unos a otros y, en algunos casos, también funcionan como adyacentes de sustantivos. En Martínez (1994a: \$ 1.3.1) puede hallarse una explicación más exhaustiva. 
un referente que quedan relegados a una función meramente expresiva. Por último, los sintagmas interjectivos parecen ir asociados sistemáticamente al contenido entonativo de la exclamación.

Si convenimos en aceptar que los sintagmas interjectivos quedan definidos por los rasgos formales señalados ${ }^{5} \mathrm{y}$, en definitiva, por su posibilidad de relación sintagmática -y solo secundariamente por su significación ${ }^{6}$ - parece claro que existe una frontera, por un lado, entre las interjecciones y los enunciados exclamativos en general; y por otro, entre la interjección y algunos grupos sintagmáticos en los que ya no cabe postular una relación interna de combinación sino de dependencia 7 . En este terreno, se detecta una falta de coincidencia en la literatura especializada a la hora de establecer los límites de la categoría interjección y sus posibilidades combinatorias. ${ }^{8}$

5 Álvarez Menéndez (2016), también desde una perspectiva funcionalista, ofrece una caracterización de los sintagmas interjectivos coincidente, en líneas generales, con nuestra propuesta. Aunque su trabajo versa sobre la lengua asturiana, la fundamentación teórica y metodológica es aplicable al español. Entre sus aportaciones, adquiere interés la delimitación de la interjección con otras unidades con las que esta clase de sintagmas comparte ciertos rasgos (vocativos, marcadores conversacionales, enunciados frásticos, fórmulas al servicio de la cortesía, etc.).

6 Constituye un aspecto básico de la teoría lingüística establecer las categorías sirviéndose de criterios funcionales, esto es, por sus posibilidades de relación sintagmática. Tal como señala Hjelmslev (1959: 188), «Si en consecuencia se pretende definir una categoría por la significación, se lleva a cabo asignándole una etiqueta semántica a una categoría establecida primero por criterios funcionales. La significación es un hecho que sólo se reconoce deductivamente, y presupone la función».

7 No cabe duda de que la definición de interjección se ajusta plenamente a las llamadas interjecciones propias y presenta más inconsistencia cuando se trata de las interjecciones impropias, pues en los casos en que estas no se comportan como incisos pasan a relacionarse como adyacentes de otra unidad con la que forman un grupo sintagmático.

8 López Bobo sostiene que la diferencia entre los sintagmas interjectivos y el resto de categorías es que los primeros no pueden formar grupo sintagmático, que son inanalizables en elementos menores, que en su interior jamás hay dependencias sintácticas y que son elementos marginales (2002: 16), aunque también reconoce que en algunos casos podría hablarse de interjecciones que llevan un adyacente subordinado ( Toma ya con el niño!) (2002: 18). En otro lugar (2002: 33), al referirse a determinadas interjecciones impropias, destaca el hecho de que puedan integrarse plenamente en estructuras exclamativas, como en los ejemplos Usted, ¿qué caramba quiere!; ; Olé la madre que te parió. Por su parte, Rodríguez Ramalle (2011) parece asumir la posibilidad de que una interjección aparezca como elemento nuclear de un grupo sintagmático en casos como ¡Vaya que sí se supo lo que ocurrió!, aun cuando precisa que «desarrollan una lectura de grado, similar a la que aparece en otras estructuras: ;Lo que sabe este cbico!, iQué de cosas sabes!, Qué pesado es este cbico!» (Rodríguez Ramalle 2011: 191). Otros autores, en cambio, separan la interjección de los enunciados exclamativos (Alonso Cortés 1999 \$ 62.4.3) o, en el caso de vaya, asimilan esta unidad a estructuras exclamativas en las que aparecen términos cuantificadores (Octavio de Toledo 2001, Casas Plaza 2004). 


\subsection{Los enunciados exclamativos}

Cualquier mensaje lingüístico establece una relación solidaria con un contenido de tipo asertivo, interrogativo o exclamativo. Este contenido es significado de un significante cuya expresión es una curva de entonación, asociada en cada caso a inflexiones tonales (o tonemas) diferenciadas. En general, resulta relevante el tonema final (anticadencia/cadencia), que en español caracteriza el significado de 'interrogación' frente al de 'aserción'. La exclamación constituiría una variedad de la aserción, «cuyo significante es una curva de entonación coincidente con la cadencia, y tal que, por su función expresiva, el contenido caracterizado no suele someterse al criterio de veracidad /falsedad» (Martínez 1994a: 250).

Así pues, los sintagmas interjectivos compartirían con los enunciados exclamativos determinados rasgos suprasegmentales en lo que atañe al significante del signo entonativo ${ }^{9}$, pero también una parte de su función externa, esto es, el hecho de que ambos están al servicio de la función expresiva del hablante:

[...] el mensaje "exclamado" se da vinculado a un referente; si, no obstante, no es frecuente que se declaren verdaderos o falsos, ello se debe a que - por su función "expresiva"se orientan sobre todo a la parte subjetiva o emotiva de la comunicación». (Martínez 1994a: 89)

Siguiendo la teoría de los actos de habla, Alonso-Cortés (1999: 3995) delimita la oración exclamativa «como un tipo gramatical de oración que corresponde a la realización de la fuerza ilocutiva del acto de habla expresivo o fuerza exclamatoria [...], marcada gramaticalmente por una clase de palabras exclamativas o palabras-cu (qué, cuál, cómo, cuánto), palabras que ponderan o intensifican algo que afecta al hablante». Reproducimos sus ejemplos de oraciones exclamativas:

(7) ¡Qué cosa más rara!

(8) ¡Si me acordaré yo!

9 El rasgo compartido más evidente es la similitud de la curva de entonación que recubre estos enunciados (Navarro Tomás 1950, Quilis 1993, Alonso-Cortés 1999: \$ 62.2). Según Quilis (1993: 453), la exclamación se caracteriza «por un descenso muy acusado del fundamental desde la primera sílaba acentuada, o por un fundamental muy bajo y plano». Por otra parte, los enunciados exclamativos comparten una estructura melódica similar a las oraciones interrogativas parciales, que presentan al inicio el nivel tonal medio (coincidiendo con el pronombre interrogativo), para finalizar con un nivel tonal bajo en cadencia. Este patrón es común, asimismo, al de las secuencias que presentan al inicio una unidad focalizada con acento enfático (Fernández Lorences 2010: 185). 
(9) ¡Qué de aceite!

(10) ¡Qué vida la suya!; ¡Qué casa esta!

Al tiempo, separa la interjección (al menos, la propia) de la oración exclamativa:

La oración exclamativa aparece acompañada de interjecciones en posición inicial. Siendo la exclamativa un acto de habla expresivo, la interjección propia concurre con aquella anticipando aspectos del significado de la oración que acompaña. (Alonso Cortés 1999: 4010)

Y ofrece el siguiente ejemplo:

(11) ¡Uf, qué cursi!

Así pues, dada esta similitud en el plano fónico entre interjecciones y enunciados exclamativos ${ }^{10}$, es preciso situar sus diferencias en otro terreno. Un criterio que se ha seguido en ocasiones es el de considerar interjecciones a los sintagmas que han perdido su primitiva significación léxica, convirtiéndose de este modo en unidades que sirven fundamentalmente a la función expresiva. En esta línea, la $N_{G L E}(\$ 32.5 . e)$ sostiene que «los límites entre interjecciones y sustantivos usados en la exclamación dependen del gado de lexicalización de las expresiones que se consideren». No obstante, es preciso tener en cuenta dos aspectos fundamentales. En primer lugar, que existen construcciones con valor cuantificativo en las que aparentes sustantivos (p. e., la tira en lee la tira de libros, o pila en estaba pila cansado), ya sin capacidad designativa, se han adaptado como cuantificadores y funcionan como adyacentes de la unidad cuantificada (San Julián Solana 2013: 42, 2015). Esta peculiaridad resulta compartida, por otra parte, por los pronombres, cuya capacidad designativa viene dada por el contexto lingüístico o situacional, pues su insuficiencia semántica los imposibilita para efectuar referencias a una clase o a una entidad existencial.

De otro lado, se ha vinculado la definición de interjección a la función expresiva, pero esta no es privativa de esta clase de sintagmas. Otras muchas construcciones que implican énfasis o intensificación permiten al emisor expresar (a veces, en el límite máximo de una escala) el grado en que el hablante

10 Quilis (1993: $\$ 14.5 .2)$ incluye dentro de las funciones de la entonación en el nivel expresivo la afirmación enfática, la pregunta pronominal enfática y la exclamación, y señala que «el patrón entonativo de la pregunta pronominal enfática coincide con el de la afirmación enfática: La distinción entre ambas entonaciones viene dada únicamente por la estructura gramatical: presencia de palabras gramaticales interrogativas frente a ausencia de las mismas» (Quilis 1993: 449). 
considera la cantidad de los objetos, propiedades o acontecimientos ${ }^{11}$. Es el caso, por ejemplo, de las oraciones consecutivas en sus diversas variantes (Álvarez Menéndez 1987, 1999; Martínez 1989): Eres tan tonto que para comer sopa de letras necesitas un traductor; Si será inconsciente, que no dudó en aceptar; ;Dice unas burradas...!; o enunciados en los que intervienen términos disfemísticos, sea en oraciones interrogativas parciales (¿Dónde cojones están mis pantalones?) sea segmentos caracterizados por la exclamación (;Qué mierda de tallarines!).

Si nos atenemos al criterio formal de que los sintagmas interjectivos se insertan como incisos en un grupo sintagmático, esta propiedad se cumple en el caso de las interjecciones propias, como sucede en el ejemplo anterior (11), pero resulta menos evidente cuando se trata de una de las llamadas impropias, lo que lleva a discutir tanto su categoría como las relaciones internas del grupo en enunciados como los siguientes:

(12) ¡Vaya con el niño!

(13) ¡Vaya reloj que te has comprado!

(14) Vaya aburrimiento...

(15) Vaya pinta tiene ese.

(16) Vaya si te conozco, pensó Teresa.

Dejando momentáneamente a un lado la discusión sobre su categoría, lo cierto es que algunos autores han señalado que en casos como los anteriores vaya es un elemento intensificador o enfático que tiene relación con la cuantificación $^{12}, y$, más en concreto, con construcciones que expresan en un grado alto una propiedad que el hablante atribuye a una entidad o a un predicado (Casas Plaza 2004, Fernández Lorences 2004, Sancho Cremades, 2008, Rodríguez Ramalle 2011, Garnes 2013, 2015, entre otros).

11 Al respecto de la cuantificación, San Julián Solana (2013: \$ 2.1) señala que «... podría quedar definida como la 'operación gramatical mediante la cual se indica de alguna forma la cantidad de los objetos, las sustancias - tanto concretos como abstractos-, las propiedades o los objetos denotados'». Esta definición, en opinión del autor, «permite incluir como parte del fenómeno cuantitativo a los recursos de comparación e incluso, tal vez, a ciertos mecanismos orientados a la consecución del énfasis».

12 Sobre esta cuestión, Casas Plaza (2004) señala: «Un tipo común en las exclamativas consiste en partir de vaya como elemento afín a una forma qu- o a adjetivos irónicos como menudo o a términos cuantificadores [...]». Recoge asimismo este valor Garnes (2013): «La forma equivalente del verbo ir también muestra un comportamiento intensificador cuando aparece seguida de sustantivos». Octavio de Toledo (2001), por su parte, ofrece un estudio diacrónico sobre la evolución de vaya y sitúa a finales del s. XviII su fijación como elemento exclamativo de grado, en enunciados del tipo ¡Vaya con las niñas del día! 
En este sentido, vaya mostraría más similitud - tanto en lo que respecta a su valor como elemento intensificador como por lo que atañe a su función como adyacente de otro sintagma - con los enunciados exclamativos en los que intervienen los relativos tónicos:

(17) a. ¡Vaya aburrimiento!

b. ¡Qué aburrimiento!

No obstante, esa afinidad en cuanto a sus propiedades ${ }^{13}$ no implica un comportamiento gramatical idéntico. En primer lugar, no existen en español relativos exclamativos como unidades diferentes de los interrogativos; estos últimos pueden convertirse en exclamativos ${ }^{14}$ (y, por lo tanto, poner de relieve la función expresiva) con la sola modificación de la curva entonativa ${ }^{15}$. Sin embargo, vaya siempre se asocia a un contenido de exclamación o aserción:

(18) a. ¿Qué coche?

b. ¡Qué coche!

(19) ¡Vaya coche! // * ¿Vaya coche?

(20) Último día de verano, y vaya día hemos tenido hoy. // ¿Vaya día hemos tenido hoy?

\section{La función de término terciario}

\subsection{Vaya que $\{$ si\} como adyacente cuantificativo de un predicado}

Si aceptamos el papel de esta unidad como un elemento intensificador de lo designado por un predicado, cabe considerar que su función en la oración es

13 Aunque nuestro propósito no es agotar aquí los paralelismos entre estas construcciones, cabe apuntar otro rasgo ligado a la intensificación: cuando funcionan como adyacentes de un sustantivo rechazan la presencia del artículo o de unidades que aporten el valor de identificación al sustantivo ( ${ }^{*}$;Vaya el aburrimiento!). Idéntico comportamiento presentan las consecutivas (Álvarez Menéndez 1989: 148 ss.).

14 Si los pronombres qué y cuán funcionan como términos terciarios, esto es, como adyacentes prenucleares de un adjetivo o un adverbio, expresan únicamente valores exclamativos (Martínez 1989: 195).

15 Véase Quilis (1993). Frente a la curva melódica de un enunciado interrogativo como ¿Qué vas a bacer?, el caracterizado por la exclamación (iQué vas a bacer!) presenta «un descenso muy acusado del fundamental desde la primera sílaba acentuada, o [...] un fundamental muy bajo y plano» (Quilis 1993: 432 y 453). 
de adyacente, y, más en concreto, su asimilación a la función que ha recibido el nombre de término terciario.

Esta función ha sido descrita con los siguientes rasgos (Martínez 1988, 1994c: 93-94, Fernández Lorences 2004): a) la unidad en función de término terciario es un adyacente de un núcleo funcionalmente adjetivo o adverbial; $b$ ) su posición sintagmática es prenuclear; c) esta función está al alcance de unidades cuyos morfemas de género, número y artículo se han de neutrilizar, de manera que no manifestan concordancia; y d) su significación es de cuantificación indefinida.

En otro lugar (Fernández Lorences 2004) hemos propuesto incluir en la función de término terciario unidades que funcionan como adyacentes oraciona$\operatorname{les}^{16}$, tales como sí que, bien que y vaya que, las cuales cumplirían estos requisitos, tanto en lo que respecta a su posición sintagmática como en lo referente a la cuantificación indefinida que aportan (gradación máxima, desde el punto de vista del emisor) o a su inmovilización morfemática.

Visto así, no cabría sostener que estamos ante interjecciones impropias que encabezan una oración introducida por la conjunción que (Rodríguez Ramalle 2007, 2011), sino que vaya que $\{s i\}$ ya se han ido soldando y configuran una unidad al servicio de la intensificación. Ello se pone de relieve si examinamos los siguientes ejemplos:

(21) El más hermoso detalle que alguien a tenido para mi es el siguiente: Mi novio me invito a acompañarlo a su examen profesional, pero ojo el vive en una ciudad bastante lejos y me pago todos los gastos, vaya que le salio caro, ya que se necesitan tomar un avion, y dos autobuses para llegar a ese lugar minimo 1 día. me ama tanto que siento que no lo merezco ${ }^{17}$. (CREA: Cosmopolitan, 04/2003: sondeo urgente); Quizá una diferencia entre los barrocos del siglo XVII y los neobarrocos de nuestros días consista en que aquéllos no sabían que eran barrocos y

16 La función de término terciario se concibe como un adyacente en el grupo nominal: una unidad de categoría adverbial que se subordina a un adjetivo o a un adverbio, y en ciertos casos, a un sustantivo, a los que cuantifica indefinidamente desde una posición sintagmática prenuclear. El hecho de de extender esta etiqueta al nivel oracional (adyacente de un predicado) no deja de entrar en contradicción con uno de sus rasgos definitorios (nivel suboracional). Sin embargo, lo cierto es que constituyen unidades que reúnen las características señaladas para esta función, por lo que parece más coherente incluirla en ella que hablar de «aditamento oracional» o «atributo oracional» (Alarcos 1970), que acogería a unidades que posteriormente se han incluido en la función incidental (Fernández Fernández 1993), tales como desgraciadamente en Desgraciadamente, todo ba terminado.

17 Así en el original. 
éstos vaya que lo saben. (CREA: Gonzalo Celorio, Ensayo de contraconquista, México D. F.: Tusquets, 2001)

(22) Me ubiqué en la vida diaria, ¡vaya si me ubiqué! (CREA: Alicia Giménez Bartlett, Serpientes en el paraíso. El nuevo caso de Petra Delicado, Barcelona: Planeta, 2002); Y vaya si te conozco, pensó Teresa. Te he visto cien veces en Sinaloa, en Melilla, en todas partes. Siempre eres el mismo. (CREA, Arturo Pérez-Reverte, La Reina del Sur, Madrid: Alfaguara, 2002).

\subsection{Supuestos núcleos que funcionan como adyacentes}

Podemos comprobar la función de vaya que \{si $\}^{\zeta}$ como núcleo o adyacente mediante las pruebas de la conmutación por cero $^{18}$ y la permutación:

(23) a. Me pagó todos los gastos, vaya que le salió caro.

b. Me pagó todos los gastos, le salió caro.

c. Me pagó todos los gastos, *vaya...

d. Me pagó todos los gastos, vaya. $(\downarrow)$

(24) a. Aquellos no sabían que eran barrocos y estos vaya que lo saben.

b. Aquellos no sabían que eran barrocos y estos lo saben.

c. Aquellos no sabían que eran barrocos y *estos vaya.

(25) a. Me ubiqué en la vida diaria, ¡vaya si me ubiqué!

b. Me ubiqué en la vida diaria, me ubiqué.

c. ?'Me ubiqué en la vida diaria, ¡vaya!

d. Me ubiqué en la vida diaria, *vaya si.

(26) a. Y vaya si te conozco, pensó Teresa.

b. Y te conozco, pensó Teresa.

c. *Y vaya (si), pensó Teresa.

18 La conmutación por cero permite comprobar la función hjelmsleviana de dependencia entre un núcleo y un adyacente: mientras el primero es capaz de mantener la función, el segundo resulta prescindible; la conmutación permite, por tanto, registrar relaciones paradigmáticas y, secundariamente, relaciones sintagmáticas. Por su parte, la permutación consiste en cambiar el orden de las unidades de la cadena sintagmática: si a este cambio de orden en el plano de la expresión le corresponde un cambio en el plano del contenido, hay que concluir que el orden de las unidades «funciona como expresión de un contenido». (Martínez 1994a: 68 ss.). 
Como podemos observar si contrastamos las secuencias de $a$ y $b$ en los ejemplos anteriores, la eliminación de vaya que $\{s i\}$ no provoca agramaticalidad: el supuesto adyacente mantiene su función oracional, solo que en estos casos el resultado es una secuencia desprovista de énfasis. En algún caso (23d), puede subsistir, pero para ello requiere un tonema final de cadencia que cambia su valor al de un inciso con el que el emisor comenta el fragmento discursivo.

Mediante esta prueba es posible comprobar asimismo que las unidades consideradas conforman un paradigma junto con otras del tipo de síque o bien que:

(27) Me pagó todos los gastos, vaya que le salió caro Me pagó todos los gastos, sí que \{́bien que ’ le salió caro / Me pagó todos los gastos, le salió caro, *vaya que.

(28) Aquellos no sabían que eran barrocos y estos vaya que lo saben Aquellos no sabían que eran barrocos y estos síque \{bien que lo saben // Aquellos no sabían que eran barrocos y *estos vaya \{que\}.

(29) Y vaya si te conozco, pensó Teresa $\sim$ Y sí que \{́bien que $\}$ te conozco, pensó Teresa / Y te conozco, vaya // Y te conozco, *vaya si.

Lo que vienen a mostrar estas pruebas es, en primer lugar, que no hay un sintagma interjectivo que funcione como núcleo de la construcción al que se subordine un adyacente (en estos casos, una oración transpuesta a categoría nominal por las conjunciones que o si), sino que, por el contrario, estamos ante una unidad compleja que funciona como adyacente con valor cuantificador. No resulta extraño en la evolución diacrónica del español la formación de locuciones conjuntivas a partir de primitivos adverbios unidos a conjunciones: aun + que > aunque, bien que ( $\approx$ aunque), como ya señalaba Andrés Bello ${ }^{19}$ (1847):

(30) Bien que hubiese grande escasez de provisiones no nos faltaba lo necesario; El camino de la derecha es llano, derecho y cómodo, bien que no le falten lozadales y ciénagas en tiempo de lluvias. (Bello 1847: $\$ 1229)$

También para Bello habría una unidad (y no dos): sí que, en sus palabras, «usado como conjunción»:

(31) Sí que hay quien tiene la hinchazón por mérito.

En segundo lugar, ha de señalarse que esta unidad (vaya que, vaya si, vaya que si) establece una relación paradigmática con otros intensificadores que, en el

19 Al referirse a estos usos, señala: «... pudiéramos poner bien que en lugar de aunque, sin hacer diferencia alguna en el sentido» (Bello 1847: \$1229). 
caso de actuar sobre el predicado, incluyen también la supuesta conjunción. Y, en tercer lugar, que la oración así intensificada contrasta con una estructura neutra o desprovista de énfasis.

\subsection{Algunos rasgos que caracterizan a esta construcción}

En primer lugar, son incompatibles con el modo subjuntivo:

(32) Vaya si le sale caro; Vaya si le salió caro; Vaya si le saldrá caro; Vaya si le saldría caro. // *Vaya si le salga caro.

Dado que son construcciones que intensifican la afirmación, rechazan el subjuntivo, asociado a valores de 'incertidumbre', tal como señala Andrés Bello (1847: $\$ 462)$ :

Piden de ordinario el subjuntivo común las palabras o frases subordinadas que denotan incertidumbre o duda, o alguna emoción del ánimo, aun de aquellas que indirectamente afirman el objeto o causa que la ocasiona [...].

Otros autores (Martins 2006, Hernanz 2007, Escandell Vidal 2011, Escandell Vidal y Leonetti 2009, 2011, García Mouton y San Segundo Cachero 2015) consideran que unidades de este tipo constituyen focos de polaridad positiva, es decir, que actúan como intensificadores de una predicación afirmativa, algo que, por otra parte, ya había sido señalado por Alarcos Llorach (1970: 333, 1994: 132) al referirse al adverbio sí (que):

Sí (que) estaba convencido de su éxito. En estos casos, sí es una unidad enfática que subraya el contenido afirmativo de la secuencia, puesto que la modalidad es afirmativa cuando no lleva indicios explícitos de lo contrario.

$\mathrm{Si}$ aceptamos que estas unidades intensifican la modalidad afirmativa, esto es, que constituyen focos de polaridad positiva, no es extraño que resulten incompatibles con los términos de polaridad negativa (Bosque 1980), como muestra el ejemplo siguiente:

(33) *Vaya si le salió caro en absoluto; *Vaya si vendrá a verte siquiera; o que rechacen una oración coordinada adversativa que incluya un término negativo:

(34) Vaya si estaba enamorado, \#pero no estaba mucho. 
Ahora bien, también admiten anteponerse al adverbio de negación no en respuestas ecoicas para intensificar una predicación recogida anafóricamente por el propio adverbio:

(35) - Eso no son cuatro centímetros, mujer.

- ¡Vaya que sí!

- ¡Vaya que no!

（36）-No lo conocerá usted.

- Vaya que no... Si he estado allí. (CREA)

En (35), el hablante emite una aseveración negativa, a la cual su interlocutor replica lo contrario para expresar desacuerdo ('Sí lo son'). En el turno de palabra siguiente, se retoma enfáticamente lo aseverado antes (;Vaya que no!). En (36) el interlocutor emite, a modo de réplica, la secuencia Vaya que no..., equivalente en este caso a una afirmación enfática ('por supuesto que sî').

\section{Variantes de la construcción con vaya como intensificador de un predicado}

A diferencia de lo que sucede cuando la unidad vaya aporta intensificación a lo referido por un sintagma de categoría nominal, cuando se antepone a un predicado suele acompañarse de las conjunciones que o si (átono), como hemos tratado de mostrar hasta aquí. Los siguientes ejemplos tomados del CREA revelan que pueden presentarse formas simples (vaya que, vaya si) (37-38) pero también combinadas (vaya que si) (39); en estas últimas una de las dos conjunciones resulta prescindible. Por su parte, los adverbios de afirmación o negación $(s i, n o)$ recogen anafóricamente del contexto anterior el contenido predicativo, por lo que es posible que se presenten precedidos de vaya que con valor intensificador (40-41). Como se ha dicho antes, en (41) el enunciado adquiere el sentido opuesto al de una secuencia negativa:

(37) Aquellos no sabían que eran barrocos y estos vaya que lo saben.

(38) ... en caso de duda, yo la viuda; lo dicen y vaya si es verdad: viudos no hay.

(39) Pues bien, Euler lo resolvió, vaya que si lo resolvió, encontró la solución general para este tipo de problemas; Euler lo resolvió, vaya si lo resolvió; Euler lo resolvió, vaya que lo resolvió. 
(40) ... enfilé hacia los servicios públicos, esperando rentabilidad de alguna forma la pasta que me descuentan todos los meses. Y lo logré, vaya que sí.

(41) - No sabrás. - Vaya que no.

\section{Hacia una caracterización del morfema de 'énfasis' en el español}

Las construcciones examinadas mantienen paralelismos evidentes con otras en las que la cadena sintagmática se inicia con una unidad focalizada, ya se trate de un foco contrastivo, de la llamada por Fernández Ramírez (1985) «anteposición con relieve», del verum focus (Escandell Vidal y Leonetti 2009, 2011; Escandell Vidal 2011) o de otras estructuras que comportan énfasis. Veamos algunos ejemplos. En primer lugar, ha de considerarse que muchas de estas construcciones al servicio de la intensificación requieren un orden de palabras determinado:

(42) Eran muy fuertes $>$ ¡Lo fuertes que eran! [NV-AD / AD-NV]

(43) Lo pasó muy mal > ¡Vaya mal que lo pasó! [NV-AD / AD-NV]

(44) Tenemos un grave problema $>i$ Menudo problema que tenemos! [NV$\mathrm{AD} / \mathrm{AD}-\mathrm{NV}]$

(45) Yo he armado un gresca en la rebotica a cuenta de Gustavo > ¡Floja gresca he armado yo en la rebotica a cuenta de Gustavo! (Fernández Ramírez, 1951c: 434) [s-NV-AD / AD-NV-s]

(46) Las empresas imperiales de España lo absorben todo entonces > Todo lo absorben entonces las empresas imperiales de España. (Fernández Ramírez, 1951C: 434) [s-NV-AD / AD-NV-s]

(47) Era tan alto, que llegaba al techo; Vaya si ganó dinero, que se compró un ático de lujo.

En estos casos, se produce una extraposición en el orden de los componentes de la cadena sintagmática (Álvarez Menéndez 1987), de manera que una parte del predicado se antepone al verbo y la secuencia sigue el patrón de [AD-V-s].

Además del orden de palabras, se precisa un elemento cuantificador que funcione como adyacente del sustantivo y que presenta lo referido por el nombre -o, en su caso, por el predicado- situado en el polo máximo de una escala cuantitativa o cualitativa. 
Cuando el elemento cuantificador es un adjetivo del tipo menudo, flojo, bonito (Hernanz 2001) y algunos otros similares, el adjetivo pierde su capacidad denotativa en favor del rasgo de 'cuantificación'; de este modo, en el contenido que se transmite opera un uso antifrástico ${ }^{20}$, pues se afirma lo contrario de lo codificado en los rasgos léxicos del adjetivo. En los enunciados recogidos en (48-50), menudo barullo o bonito lío no implican un barullo 'menudo' o un lío 'bonito', como sucedería si su papel adjetival fuese el de subclasificar o destacar alguna cualidad del sustantivo. Por otra parte, el hecho de que el valor intensificador que encierran estas secuencias esté sujeto irremisiblemente a determinado orden de palabras queda de manifiesto cuando este se altera, puesto que se originan secuencias agramaticales:

(48) ¡Menudo barullo había! // *Había barullo menudo.

(49) ¡En bonito lío me he metido! //*Me he metido en bonito lío.

(50) ¡Vaya reloj que te has comprado //*Te has comprado vaya reloj.

El contenido enfático de (48-49) resulta equivalente al de (50), en que aparece vaya como elemento intensificador. Con todo, hay que advertir que cuando vaya focaliza un predicado acentuando su valor asertivo esta unidad admite ir interpuesta entre el tema y el comentario, de manera que la inversión que resulta obligada cuando actúan como adyacentes de un grupo nominal no constituye en estos casos un requisito imprescindible. En consecuencia, pueden originarse secuencias que presentan distinta distribución sintagmática, pues en unos casos el tema queda en posición extrapredicativa (Fernández Lorences 2010: $\$ 3.1 .7$ ) y el énfasis solo afecta al predicado (51), mientras que en otros el elemento intensificador ocupa la primera posición de la cadena sintagmática y su ámbito abarca no solo el predicado sino también el tema (52):

(51) A ellas, vaya si les interesaba conocer los detalles [TEMA - (INT > PRED)]

(52) Vaya si a ellas les interesaba conocer los detalles [INT > (TEMA - PRED)]

Lo cierto es que estos elementos suponen estrategias de focalización que inciden en la polaridad de la oración. Así concebido, el énfasis constituiría el resultado del foco en la polaridad que marcaría abiertamente la aserción de un contenido proposicional, de manera que este se asevera «de manera muy fuerte» (Leonetti y Escandell Vidal 2008, 2009).

20 Agradezco a Aurelio González Ovies su observación sobre la posible presencia de una antífrasis en algunos usos que implican cuantificación y, en ocasiones, ironía. 
Ahora bien, dados los paralelismos con otras construcciones - algunas de las presentadas aquí de manera escueta y otras que podrían añadirse- también cabría postular que el español alberga en su estructura gramatical un morfema $^{21}$ de 'énfasis', de modo que estos procedimientos de intensificación ${ }^{22}$ no resultan dependientes del contexto de enunciación, esto es, conforman estructuras lingüísticamente codificadas.

Así concebido, el énfasis constituiría una figura de contenido de carácter extenso (caracteriza no solo al lexema verbal sino también a los demás componentes de la oración) y mixto (puede establecer a la vez relaciones entre sintagmas de la misma oración y entre sintagmas de oraciones diferentes). Aunque esta figura de contenido no tiene por qué corresponderse de manera biunívoca con una forma estable en el plano de la expresión, se expresaría mediante algunos elementos recurrentes: orden de palabras, curva melódica y presencia de unidades cuantificadoras, componentes que oponen las secuencias neutras o desprovistas de énfasis a aquellas otras en las que opera este morfema.

\section{Conclusiones}

5.1 La interjección constituye una categoría de sintagmas definida por sus posibilidades de relación sintagmática: $a$ ) no puede entrar en relaciones de combinación o subordinación ni con sintagmas de otras categorías ni con los de su misma categoría; $b$ ) su capacidad de relación queda limitada a una mera yuxtaposición (no coordinable) como inciso incrustado en un grupo sintagmático de categoría nominal o verbal.

5.2 Las interjecciones son signos autónomos mínimos en cuyo interior no resulta posible separar rasgos léxicos ni morfológicos. Su misión consiste en servir a la función expresiva del hablante. Están generalmente asociadas a la modalidad entonativa de la exclamación.

5.3 Los enunciados exclamativos comparten con la interjección determinados rasgos suprasegmentales y una parte de su función externa. A su vez, la función expresiva se une a la referencial en otras construcciones del español que conllevan énfasis o intensificación (oraciones consecutivas, interrogativas focalizadas o exclamativas en las que aparecen términos disfemísticos).

21 La gramática funcional del español define el morfema como una figura de contenido gramatical (Martínez 1994a), esto es, una parte del signo del plano del contenido.

22 Quedarían excluidos, claro está, aquellos recursos de intensificación en los que intervienen únicamente rasgos léxicos (Era una máquina; Se portó como un cabronazo, Me gustó mucho, mucho...). 
5.4 La insuficiencia semántica que caracteriza a las interjecciones no es privativa de estas: aparece también en primitivos sustantivos adaptados ya como adverbios, o en los pronombres, cuya capacidad designativa viene dada por el contexto lingüístico o situacional.

5.5 La forma vaya puede aparecer para intensificar lo designado por un predicado. En estos casos, requiere ir acompañada de una primitiva conjunción (que, si) con la que ya forma una unidad. No estamos, pues, ante una interjección que actúa como núcleo al que se subordina una oración transpuesta a categoría sustantiva. Por el contrario, determinadas pruebas formales muestran que la supresión de la supuesta interjección no origina una secuencia agramatical sino un enunciado desprovisto de énfasis.

5.6 Los paralelismos que se detectan con otras construcciones en las que aparecen focos o elementos cuantificadores y que originan inversión del orden de palabras neutro permiten formular la hipótesis de que el español podría albergar en su estructura gramatical un morfema de 'énfasis' que, en el plano de la expresión, se configuraría en determinado orden de palabras, ciertos rasgos suprasegmentales y la presencia de elementos cuantificadores o intensificadores.

\section{Bibliografía}

Alarcos Llorach, E. (1970): Estudios de gramática funcional del español. Madrid: Gredos.

Alarcos Llorach, E. (1994): Gramática de la Lengua Española. Madrid: EspasaCalpe.

Alonso-Cortés, Á. (1999): «Las construcciones exclamativas. La interjección y las expresiones vocativas». En: Ignacio Bosque, Violeta Demonte (dirs.), Gramática descriptiva de la lengua española. Madrid: Espasa-Calpe, 39934050.

Álvarez [Menéndez], A. I. (1987): «Énfasis y transposición de oración a sustantivo». Revista Española de Lingüística, 17, 2, 347-370.

Álvarez [Menéndez], A. I. (1989): Las construcciones consecutivas en español. Estudio funcional sobre la oración compuesta. Oviedo: Departamento de Filología Española.

Álvarez [Menéndez], A. I. (1999): «Las construcciones consecutivas». En: Ignacio Bosque, Violeta Demonte (dirs.), Gramática descriptiva de la lengua española. Madrid: Espasa-Calpe, 3739-3804. 
Álvarez [Menéndez], A. I. (2016): «Caracterización funcional de la interxección: a propósitu de delles interxecciones del asturianu». Revista de Filoloxía Española, 16, 9-48.

Bello, A. (1847): Gramática de la lengua española destinada al uso de los americanos (con las notas de Rufino José Cuervo) (est. y ed. Ramón Trujillo). Madrid: Arco/Libros, 1988.

Bosque, I. (1980): Sobre la negación. Madrid: Cátedra.

Carbonero Cano, P. (1990): «Configuración sintáctica de los enunciados exclamativos». Pbilologia Hispaliensis, 5, 111-138.

Casas Plaza, A. (2004): «Exclamativas y expelitividad. El que enfático». Revista de Filología Española, 84, 2, 265-283.

Escandell Vidal, M. V. (2011): «Verum focus y prosodia: cuando la duración (sí que) importa». Oralia, 14, 181-202.

Escandell Vidal, M. V., Leonetti Jungl, M. (2009): «La expresión del verum focus en español». Español Actual, 92, 11-46.

Escandell Vidal, M. V., Leonetti Jungl, M. (2011): «Bastantes problemas ( ${ }^{*}$ no) tenemos...». En: M. Victoria Escandell Vidal, Manuel Leonetti, Cristina Sánchez López (eds.), 60 Problemas de gramática: dedicados a Ignacio Bosque. Madrid: Akal, 253-260.

Fernández Fernández, A. (1993): La función incidental en español. Hacia un nuevo modelo de esquema oracional. Oviedo: Departamento de Filología Española.

Fernández Lorences, T. (2004): «Eso sí que no se puede negar: la intensificación con términos terciarios». Interlingüística, 15, 1, 487-496.

Fernández Lorences, T. (2010): Gramática de la tematización en español. Oviedo: Ediuno.

Fernández Ramírez, S. (1985): Gramática española (ed. Ignacio Bosque, José Polo). Madrid: Arco/Libros, vol. I.

García Mouton, P., San Segundo Cachero, R. (2015): «La polaridad negativa en los datos asturianos del ALPI». En: Ramón d'Andrés Díaz, Taresa Fernández Lorences, Juan Carlos Villaverde Amieva (eds.), Varia asturlleonesa. N'bomenaxe a José A. Martínez. Uviéu: Trabe, 53-78.

Garnes, I. (2015): «Desarrollo de intensificadores con origen verbal en el español coloquial del siglo XIX». Études Romanes de Brno, 36, 2, 67-85. 
González Rodríguez, R. (2007): «Sintaxis y semántica de la partícula de polaridad sì. Revista Española de Lingüística, 37, 311-36.

Hernanz, M. L. (2001): «iEn bonito lío me he metido!: notas sobre la afectividad en español». Moenia, 7, 93-109.

Hernanz, M. L. (2007): «From polarity to modality. Some (a)symmetries between bien and sí in Spanish». En: Luis Eguren, Olga Fernández Soriano (eds.), Coreference, Modality, and Focus. Studies on the Syntax-Semantics interface. Amsterdam: John Benjamins, 133-170.

Hjelmslev, L. [1943]: Prolegómenos a una teoría del lenguaje (v. esp. José Luis Díaz de Liaño). Madrid: Gredos, 19842.

Hjelmslev, L. [1959]: Ensayos lingüísticos (trad. esp. Elena Bombín Izquierdo, Félix Piñero Torre). Madrid: Gredos, 1972.

Leonetti, M., Escandell Vidal, V. (2008): «Las anteposiciones inductoras de foco de polaridad». En: Antonio Moreno Sandoval (ed.), Actas del VIII Congreso de Lingüística General. Madrid: Universidad Autónoma de Madrid.

Leonetti, M., Escandell Vidal, V. (2009): La expresión del verum focus en español». Español Actual, 92, 11-46.

López Bobo, M. J. (2002): La interjección: aspectos gramaticales. Madrid: Arco/ Libros.

Martins, A. M. ${ }^{a}$ (2006): «Emphatic affirmation and polarity». En: Jenny Doetjes, Paz González (eds.), Romance Languages and Linguistic Theory 2004. Amsterdam: John Benjamins, 197-223.

Martínez, J. A. (1988): «Sobre una construcción del español, que son dos». Verba, 15, 265-287.

Martínez, J. A. (1994a): Propuesta de gramática funcional. Madrid: Istmo.

Martínez, J. A. (1994b): Funciones, categorías y transposición. Madrid: Istmo.

Martínez, J. A. (1994c): Cuestiones marginadas de gramática española. Madrid: Istmo.

Martínez, J. A. (1989): El pronombre II. Numerales, Indefinidos y Relativos. Madrid: Arco/Libros.

Navarro Tomás, T. (1950): Manual de pronunciación española. Madrid: CSIC.

Octavio de Toledo, Á. S. (2001): «نUn viaje de ida y vuelta? La gramaticalización de vaya como marcador cuantificador». Anuari de Filología, 11, 47-72. 
Quilis, A. (1993): Tratado de fonología y fonética españolas. Madrid: Gredos.

Real Academia Española (2014): Diccionario de la lengua española, 23 $3^{\mathrm{a}}$ ed. Madrid: Espasa.

Real Academia Española y Asociación de Academias de la Lengua Española (2009): Nueva gramática de la lengua española. Madrid: Espasa.

Rodríguez Ramalle, T. M. (2007): «Las interjecciones llevan complementos, ¡vaya que sí!». Español Actual: Revista de Español Vivo, 87, 111-125.

Rodríguez Ramalle, T. M. (2011): «La expresión del grado en las interjecciones y la función de la conjunción que». Verba, 38, 191-217.

San Julián Solana, J. (2013): Los numerales sustantivos en español. Oviedo: Ediuno.

San Julián Solana, J. (2014): «La impronta del funcionalismo en los estudios lingüísticos del asturiano». Revista de Filoloxía Asturiana, 14, 15-51.

San Julián Solana, J. (2015): La expresión sustantiva de la cuantificación en español [tesis doctoral]. Oviedo: Universidad de Oviedo.

Sancho Cremades, P. (2008): «La sintaxis de algunas construcciones intensificadoras en español y en catalán coloquiales». Verba. Anuario Galego de Filoloxía, 35, 199-233. 
Taresa Fernández Lorences

University of Oviedo

\section{Emphasis and word order in constructions with vaya in Spanish language}

Key words: interjection, tertiary term, focus, emphasis, intensification, grammar

The Spanish subjunctive form vaya historically split into different form and has given rise to units of different categories which show different syntactic behavior, from the interjection (iVaya!) to more or less fixed expressions in which the original movement meaning of the verb ir has bleached into those of doubt or desire (Deja que se vaya > Vaya con Dios, Vaya por Dios, Vaya usted a saber...).

Alongside these forms, there are other sequences whose grammatical boundary with the interjections has not always been clearly delineated: ;Vaya mierda de nudos!; Vaya susto que me has dado...; Lo dicen y vaya si es verdad. Are we then dealing with an interjection combined with an adjacent unit or, on the other hand, are vaya or vaya si acting as intensifiers of the syntagmatic group to which they belong?

We here defend the latter hypothesis with a special focus on those cases in which vaya, is morpohologically frozen and appears to act as an intensifier of a predicate or, in different contexts, a noun. Using a Functional Grammar approach, we examine the grammatical properties of constructions such as Vaya si es verdad; Aquellos no lo sabian y estos vaya que lo saben demand (in particular, suprasegmental features and word order), their syntactic variants and their similarities with other emphatic constructions involving stressed relatives or quantifiers. 


\section{Taresa Fernández Lorences}

Univerza v Oviedu

\section{Poudarek in besedni red v nekaterih strukturah z vaya}

Ključne besede: medmet, término terciario, fokus, poudarek, poudarjanje, slovnica

V španščini je oblika subjunktiva vaya skozi zgodovinski razvoj jezika pridobivala raznovrstne vloge in spodbudila nastanek enot raznih besednih vrst z različnimi skladenjskimi značilnostmi, ki segajo od medmetne rabe ( Vaya!) do bolj ali manj ustaljenih izrazov, v katerih je pomen premikanja, sicer lasten glagolu ir, nadomestilo izražanje dvoma ali želje (Deja que se vaya > Vaya con Dios, Vaya por Dios, Vaya usted a saber ...).

Poleg omenjenih poznamo tudi strukture, ki jih ne moremo vedno jasno slovnično ločiti od medmetov: ;Vaya mierda de nudos!; Vaya susto que me bas dado ...; Lo dicen y vaya si es verdad. Gre torej za »neprave« medmete s podrednim določilom, lahko pa, nasprotno, vaya in vaya si opredelimo kot podredni določili v vlogi ojačevalcev oz. poudarjalcev sintagmatske zveze, ki se ji podrejata in katere del tvorita.

Pričujoča študija zagovarja drugo možnost, pri čemer se osredotoča na primere, kjer se morfemsko nepregibni vaya, ki je izgubil vsakršen leksikalni pomen premikanja, uporablja za intenzivno poudarjanje katere izmed lastnosti označenega $\mathrm{v}$ povedku ( $\mathrm{v}$ drugih kontekstih pa označenega v samostalniku). V skladu s teoretskimi in metodološkimi načeli funkcionalne slovnice španskega jezika avtorica preučuje slovnične pogoje, ki jih zahtevajo strukture, kot sta Vaya si es verdad; Aquellos no lo sabian y estos vaya que lo saben (predvsem kar zadeva suprasegmentalne vidike in besedni red), njihove skladenjske različice ter podobnosti in razlike $\mathrm{z}$ drugimi poudarjalnimi strukturami, $\mathrm{v}$ katerih se pojavljajo naglašeni oziralni zaimki ali količinski prislovi ter samostalniški in pridevniški zaimki (Fernández Ramírez, 1985; Fernández Lorences, 2004, 2010; Casas, 2004; Leonetti y Escandell Vidal, 2008, 2009; Escandell Vidal y Leonetti 2009, 2011; Rodríguez Ramalle, 2011). 